\title{
Schnelle Rasterkraftmikroskopie durch moderne Regelungstechnik und mechatronische Systemintegration
}

J. Steininger, S. Kuiper, S. Ito, G. Schitter

Für die Rasterkraftmikroskopie (AFM) ist eine schnelle und hochpräzise Führung der AFM-Positioniereinheit und Messspitze ausschlaggebend. Besonders bei hohen Scangeschwindigkeiten führt die Dynamik der AFM-Positioniereinheit zu Abbildungsartefakten, wodurch die maximale Messgeschwindigkeit und Bildqualität eingeschränkt wird. In diesem Artikel werden moderne Ansätze diskutiert, welche zu einer signifikanten Steigerung der Messgeschwindigkeiten führen.

Schlüsselwörter: Rastersondenmikroskopie; Rasterkraftmikroskop; Regelungstechnik; Nanotechnologie; Nanometrologie

High-speed atomic force microscopy by modern control and mechatronic system integration.

In atomic force microscopy (AFM) high-performance and high precision control of the scanning-system is crucial. At high imaging speeds the dynamic behaviour of the scanner may cause imaging artefacts limiting the maximum imaging rate. This contribution discusses recent improvements for faster imaging by utilizing modern mechatronic and control engineering methods.

Keywords: SPM; AFM; scanning probe; control; nanotechnology; nanometrology

\section{Einführung}

Die Rasterkraftmikroskopie (engl. atomic force microscopy, AFM) (Binnig, Quate, 1986) stellt in der Mikro- und Nanotechnologie ein grundlegendes Verfahren zur Charakterisierung von Oberflächen auf der Nanometerskala dar. Die Methode ermöglicht die Oberflächenanalyse mit atomarer Auflösung, wobei sie ebenso die Bestimmung einer Vielzahl von physikalischen und chemischen Materialeigenschaften erlaubt. Hierzu zählen, neben der eigentlichen Topographie, unter anderem die Rauigkeit, Adhäsion, Phasenübergänge, elektrische und magnetische Feldverteilungen, Dichteverteilungen und vieles mehr.

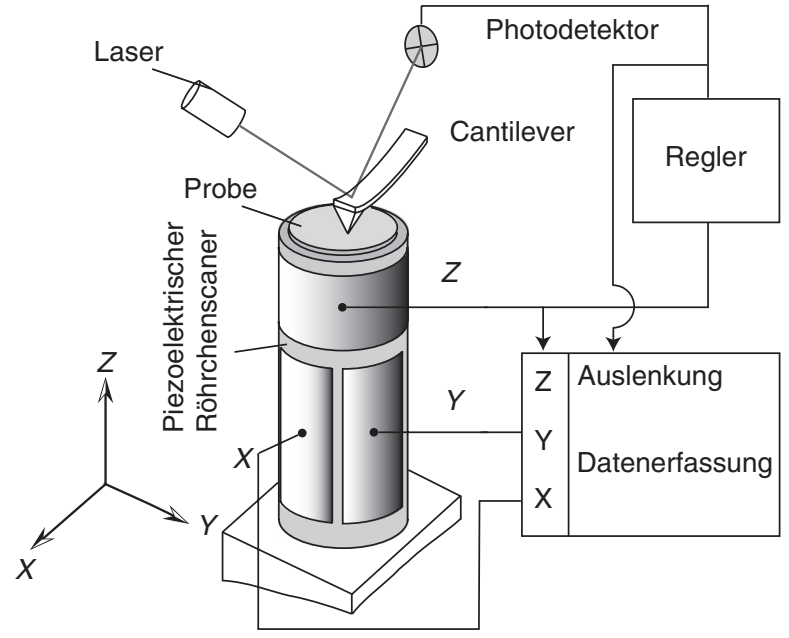

Abb. 1. Funktionsprinzip eines Rasterkraftmikroskops mit einem piezoelektrischen Röhrchenscanner
Das Messprinzip beim AFM beruht auf dem Abtasten der Oberfläche mit einer Messspitze, welche einen Spitzenradius von wenigen Nanometern aufweist. Wie in Abb. 1 dargestellt, befindet sich die Spitze am freien Ende eines mikromechanischen Federbalkens (engl. Cantilever), wobei die intermolekularen Kräfte zwischen Probe und Spitze eine Auslenkung des Cantilevers sowie Torsion durch Scherkräfte bewirken. In einer der möglichen Betriebsarten, dem so genannten "Constant Force Contact Mode", wird die Auslenkung des Cantilevers durch die intermolekularen Kräfte erfasst. Während der lateralen Bewegung der Spitze über der Probe (Scannen) wird die Auslenkung des Cantilevers durch eine vertikale Abstandsänderung in einer geschlossenen Regelschleife konstant gehalten, weshalb die Rasterkraftmikroskopie zu den kompensierenden Messverfahren zählt. Die Erfassung der Biegung und Torsion des Cantilevers erfolgt meist mittels Laserlichtzeiger (Alexander et al., 1989), wobei die Auslenkung des Cantilevers zur Ablenkung eines Laserstrahls führt, welche über einen Photodetektor gemessen wird. Da die Auslenkung, und somit die intermolekulare Kraft zwischen Probe und Cantilever, durch die Regelung konstant gehalten wird, kann durch Aufzeichnung der Stellbewegung als Funktion der Cantilever-Position während des Scanvorgangs die Topographie der Probenoberfläche rekonstruiert werden.

Eine wesentliche Einschränkung beim Einsatz von AFMs stellt die Messgeschwindigkeit dar, wodurch beispielsweise in der

Steininger, Jürgen, Dipl.-Ing., Technische Universität Wien, Institut für Automatisierungs- und Regelungstechnik, Gußhausstraße 27-29, 1040 Wien, Österreich; Kuiper, Stefan, M.Sc., Delft University of Technology, Delft Center for Systems and Control, Mekelweg 2, 2628 CD, Delft, Niederlande; Ito, Shingo, M.Sc., Schitter, Georg, Univ.-Prof. Dipl.-Ing. Dr. sc. techn. Dipl. NDS ETHZ, Technische Universität Wien, Institut für Automatisierungs- und Regelungstechnik, Gußhausstraße 27-29, 1040 Wien, Österreich (E-Mail: schitter@acin.tuwien.ac.at) 
Qualitätssicherung der Halbleiterindustrie der Durchsatz durch diese Geräte eingeschränkt wird. Ebenso ist eine direkte Beobachtung von molekularen Prozessen mit dem AFM durch die limitierte Abbildungsgeschwindigkeit nicht möglich (Hansma et al., 2006). Die laterale Geschwindigkeit wird dabei vor allem durch die mechanischen Resonanzen des Scanners begrenzt. Die vertikale Geschwindigkeit wird durch die Bandbreite der Regelschleife bestehend aus Cantilever, Messsystem zur Auslenkung, Regler, Leistungsverstärker und Mechanik inklusive Aktuator zur Positionierung begrenzt. Viele marktübliche AFMs verwenden, wie in Abb. 1 dargestellt, einen piezoelektrischen Röhrchenscanner, welcher sowohl die laterale als auch vertikale Positionierung ermöglicht (Binnig, Smith, 1986).

In diesem Artikel werden Methoden zur Erhöhung der Messgeschwindigkeit und Verbesserung der Abbildungsqualität in der Rasterkraftmikroskopie vorgestellt. Im ersten Teil wird zur Dämpfung der Resonanzen der lateralen Scaneinheit die Nutzung des so genannten Piezo self-sensing-Effekts präsentiert. Im zweiten Teil wird die duale Aktuation der vertikalen Positioniereinheit zur Erhöhung der Bandbreite diskutiert.

\section{Lateral - Self-Sensing Piezo}

Zur Abbildung der Oberfläche wird die Messspitze des Rasterkraftmikroskops in dreieckförmigen lateralen Scanbewegungen mit möglichst konstanter Geschwindigkeit über die Probe geführt (Sarid, 1994). Bei schnellen Scans regen die hochfrequenten spektralen Anteile der Scanbewegung die erste schwach gedämpfte mechanische Resonanz der Positioniereinheit an, was in weiterer Folge zu Abbildungsfehlern des AFMs führt (Croft, Shed, Devasia, 2001; Schitter, Stemmer, 2004). Durch eine starke mechanische Kopplung der Aktuatoren treten die Resonanzen und Abbildungsfehler nicht nur in der hochfrequent aktuierten Achse auf, sondern regen durch mechanisches Übersprechen Resonanzen in allen Raum- und Aktuationsrichtungen an (Rifai, Youcef-Toumi, 2001). Eine Reduzierung dieser unerwünschten Effekte wird beispielsweise durch eine sinusförmige Scanbewegung erreicht (Picco et al., 2007), welche jedoch durch die komplexen nichtlinearen Effekte beim Messen der Oberflächentopographie zu einem erheblichen Mehraufwand im PostProcessing und einer äußerst erschwerten Interpretation der Daten führt.

Eine weitere Möglichkeit stellt die Kompensation der Resonanzen durch den Einsatz einer Vorsteuerung beziehungsweise von Filter dar. Eine Ansteuerung ohne Rückführung der aktuellen Auslenkung des Aktuators (open loop) ist jedoch nur bei der genauen Kenntnis der dynamischen Systemeigenschaften hinreichend (Croft, Shed, Devasia, 2001; Schitter, Stemmer, 2004). Auch wenn eine Charakterisierung des Systems gegebenenfalls möglich ist, kann die Dynamik im Allgemeinen nicht als konstant betrachtet werden, da beispielsweise eine Massen- und/oder Schwerpunktsänderung durch einen Wechsel der Probe zu einer Veränderung der dynamischen Systemeigenschaften führt.

Abhilfe schafft hier der Einsatz von geschlossenen Regelkreisen (closed loop), welche in bestimmten Grenzen unempfindlich gegenüber Dynamikänderungen der Regelstrecke sind (Tamer, Dahleh, 1994; Salapaka et al., 2002; Schroeck, Messner, 1999). Die robuste aktive Dämpfung von Resonanzen durch den Einsatz eines geschlossenen Regelkreises erfordert jedoch die Messung der tatsächlichen Längenänderung des Aktuators (Tamer, Dahleh, 1994; Bhikkaji et al., 2007). Hierbei ist vor allem das Messrauschen in Bezug auf die Sensitivität der Messeinrichtung zu beachten, da dieses die erreichbare Mess- und somit Regelbandbreite bestimmt. Da der Einsatz von dezidierten Sensoren mit der erforderlichen Auflösung und Bandbreite in vielen Fällen aus Kostengründen nicht umsetzbar ist, wird im Folgenden eine Lösung vorgestellt, welche eine Eigenschaft der Aktuatoren zur Messung ihrer Längenänderung nutzt.
Piezoelemente zeigen den piezoelektrischen Effekt (Gleichung 1), welcher einerseits beim Anlegen einer elektrischen Spannung $u_{p} z u$ einer Änderung der Länge $x$ des Aktuators führt und andererseits beim Einwirken einer externen Kraft $f_{p}$ auf den Aktuator eine Potentialdifferenz $u_{p} z$ wischen den Elektroden hervorruft. Bestimmend ist dabei die piezoelektrische Konstante $d$, die Steifigkeit k sowie die Kapazität $C_{p}$, in welcher die Ladung $q$ gespeichert wird. Die Effekte finden meist unabhängig voneinander in konventionell erhältlichen Sensoren oder Aktuatoren Anwendung.

$$
\left[\begin{array}{l}
x(t) \\
q(t)
\end{array}\right]=\left[\begin{array}{cc}
k^{-1} & d \\
d & C_{p}
\end{array}\right]\left[\begin{array}{l}
f_{p}(t) \\
u_{p}(t)
\end{array}\right]
$$

Die hier vorgestellte Methode vereint die Nutzung beider Effekte und ermöglicht die simultane Aktuation und Längenmessung durch das Piezoelement (Dosch, Inman, Garcia, 1992; Fleming, Moheimani, 2006; Kuiper, Schitter, 2010). Dazu wird das Piezoelement in einer kapazitiven Messbrücke betrieben (Abb. 2), bei welcher

$$
\frac{C_{1}}{C_{2}}=\frac{C_{p}}{C_{s}}
$$

ist, wobei $C_{p}$ die Kapazität des piezoelektrischen Wandlers darstellt.

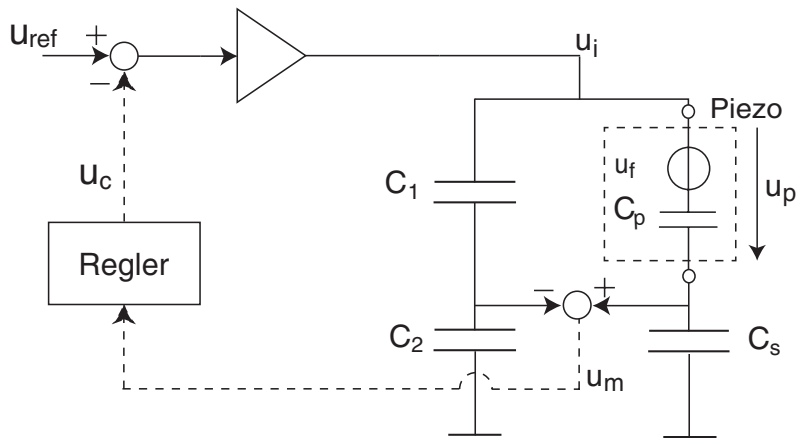

Abb. 2. Brückenschaltung zur gleichzeitigen Aktuation und Messung (Self-Sensing) mit einem Piezoelement

Eine Veränderung der Spannung $u_{i}$ führt dabei zu einer proportionalen Spannungsänderung am Piezoelement, welches sich als Aktuator im AFM entsprechend dehnt oder verkürzt, wodurch die Positionierung in allen Raumrichtungen ermöglicht wird. Die reaktive Kraft, welche durch die vom Piezoelement bewegte Masse $m$ auf den Aktuator wirkt, führt zur Differentialgleichung

$$
f_{p}=-m \ddot{x}(t)-\beta \dot{x}=k\left(x(t)-d \cdot u_{p}(t)\right),
$$

wobei $\beta$ die meist vernachlässigbare Dämpfung der mechanischen Struktur repräsentiert. Die Laplace-Transformation der Gleichung 3 führt mit der Eigenfrequenz $\omega_{n}=\sqrt{\mathrm{k} / \mathrm{m}} \mathrm{zu}$

$$
\frac{U_{p}(s)}{X(s)}=\frac{k}{d}\left(\frac{s^{2}}{\omega_{n}^{2}}+\frac{\beta}{k} s+1\right),
$$

woraus folgt, dass die kraftinduzierte Spannungsdifferenz am Piezoelement für eine kleine Dämpfung $\beta$ beziehungsweise für hohe Frequenzen $\omega \gg \beta / k$ proportional der Beschleunigung der Masse ist (Kuiper, Schitter, 2009). Somit wird eine gleichzeitige Verwendung als Sensor ermöglicht.

Ohne den zusätzlichen Einsatz von dezidierten Positionssensoren kann, alleine durch Änderungen im elektronischen Design, die Längenänderung und externe Kraftweinwirkung erfasst, und wie in Abb. 2 dargestellt, einem Regler zur aktiven Dämpfung zugeführt werden. 

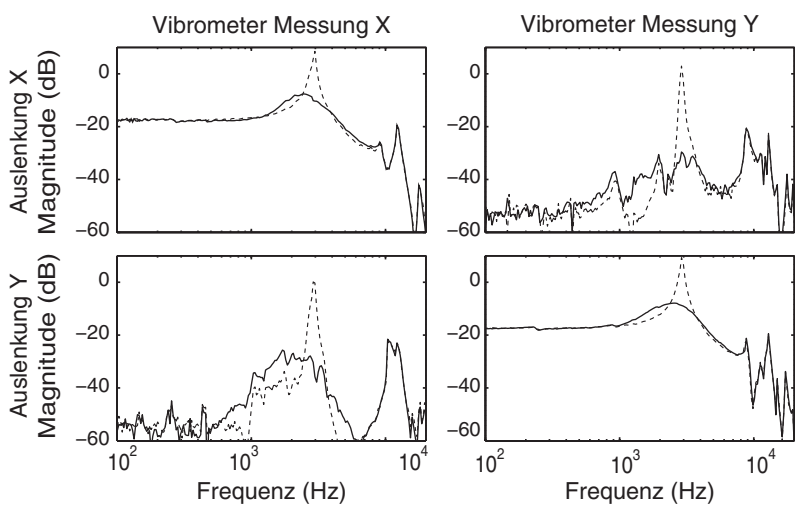

Abb. 3. Frequenzgang des Röhrchenscanners mit und ohne (strichliert) aktive elektronische Dämpfung, gemessen mit einem Vibrometer

Die Abb. 3 zeigt den Frequenzgang eines Röhrchenscanners gemessen mit dem Vibrometer OFV-5000 (Firma Polytec, Waldbronn, Deutschland). Die strichlierte Linie stellt dabei den Frequenzgang ohne, die durchgezogene Linie den Frequenzgang mit aktiver Dämpfung dar.

Ohne aktive Dämpfung (strichlierte Linie) ist in beiden Scan-Richtungen $(X, Y)$ die mechanisch schwach gedämpfte Resonanz bei $3 \mathrm{kHz}$ deutlich zu erkennen. Zusätzlich zeigt sich ein starkes Übersprechen zwischen den lateralen Richtungen, wodurch es zu einer Anregung der jeweils orthogonal zur Aktuationsrichtung liegenden Resonanzen kommt. Durch die aktive Dämpfung der Resonanzüberhöhung bei $3 \mathrm{kHz}$ kann diese um $18 \mathrm{~dB}$ verringert und gleichzeitig das Übersprechen zwischen den beiden Achsen um $30 \mathrm{~dB}$ reduziert werden.

Die Verbesserung der Abbildungsqualität des AFMs durch die aktive Dämpfung ist in Abb. 4 zu sehen. Dabei wurde ein Kalibrationsgitter mit einem Gitterabstand von $476 \mathrm{~nm}$ bei einer Zeilenfrequenz von $122 \mathrm{~Hz}$ mit und ohne aktive Dämpfung des
Röhrchenscanners gemessen. Dabei stellt die Topographie die tatsächlich durchgeführte Kompensationsbewegung des Rasterkraftmikroskops in vertikaler Richtung dar. Die Auslenkung des Cantilevers repräsentiert die verbleibende Regelabweichung. Zusätzlich wird die Reibung zwischen der AFM-Messspitze und der Probe bei konstanter Abbildungskraft aus der Torsion des Cantilevers abgeleitet.

Die Abb. 4a) bis c) zeigen deutliche Abbildungsfehler, welche durch Oszillationen der ersten schwach gedämpften mechanischen Resonanz des Röhrchenscanners entstehen. Durch die aktive Dämpfung des Röhrchenscanners können diese Oszillationen, wie in Abb. 4d) bis f) gezeigt, weitestgehend eliminiert werden. Die Abbildungsqualität kann ohne Änderungen am mechanischen Aufbau, lediglich durch Anpassungen des elektronischen Designs zur aktiven Dämpfung des self-sensing Piezos, signifikant verbessert werden

\section{Vertikal - dual aktuiert}

Neben der im vorhergehenden Abschnitt besprochenen lateralen Dynamik stellt die Regelungsbandbreite der Kraftnachführung zur Messung der Probentopographie die Beschränkung der Messgeschwindigkeit des AFMs dar. Die vertikale Bandbreite wird durch den mechanischen Aufbau, den Cantilever, die Messeinrichtung, den elektronischen Regler, die Leistungsverstärker sowie die verwendeten Aktuatoren bestimmt. Eine möglichst hohe Bandbreite dieses Regelkreises soll dabei die vertikale Auslenkung des Cantilevers, welche der verbleibenden Regelabweichung entspricht, minimieren. Besonders höhere laterale Geschwindigkeiten der AFM-Spitze über der Probenoberfläche erfordern eine höhere vertikale Bandbreite, um der Probentopographie zu folgen.

Die in vielen AFMs eingesetzten piezoelektrischen Röhrchenscanner (Abb. 5a) begrenzen meist aufgrund ihrer Bauform, und den damit verbundenen niederfrequenten und schwach gedämpften Resonanzen, die Bandbreite des vertikalen Regelkreises. Beim Einsatz von entsprechend kleinen piezoelektrischen Stack- oder Platten-Aktuatoren treten erst bei deutlich höheren Frequenzen mechanische
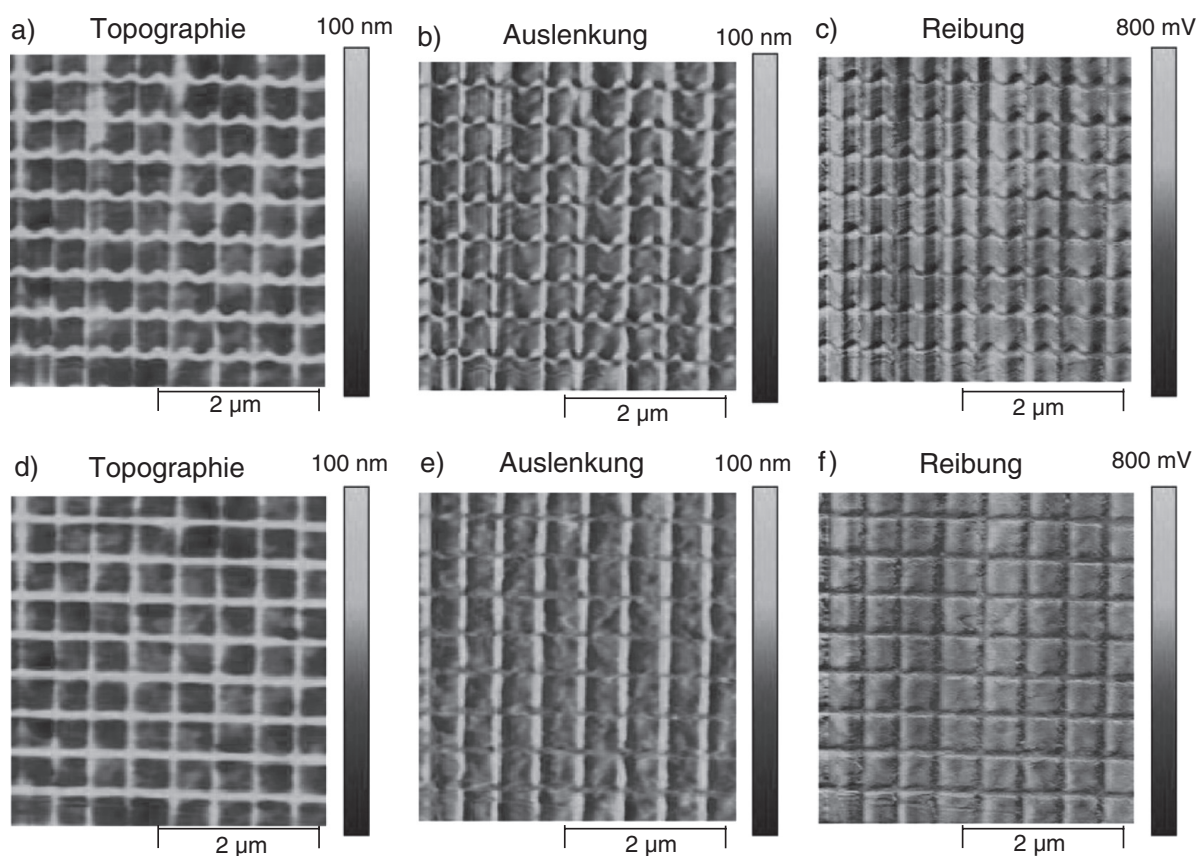

Abb. 4. Reduktion von Bildartefakten durch Scanneroszillationen (a-c) bei hoher Scangeschwindigkeit durch eine self-sensing-basierte aktive Dämpfung $(d-f)$. Die Abbildungsqualität des quadratischen Referenzgitters mit einer Periode von $476 \mathrm{~nm}$ wird durch die aktive Dämpfung signifikant gesteigert 

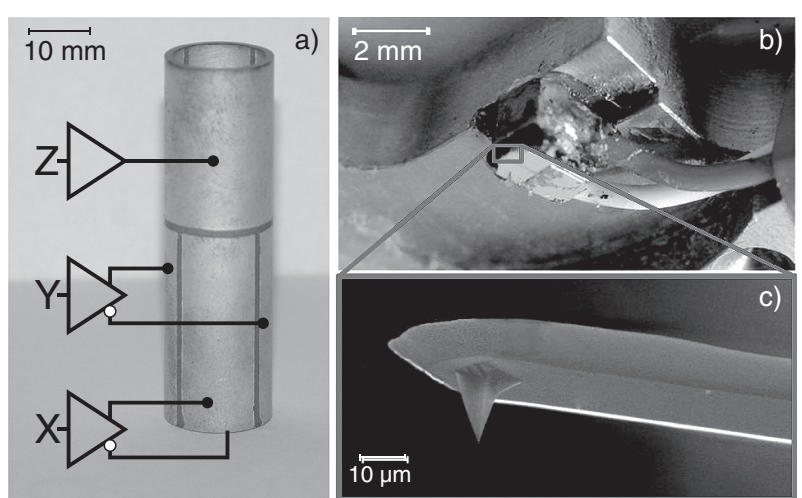

Abb. 5. (a) Piezo Röhrchenscanner für X-, Y- und Z-Aktuation, (b) Piezoelektrischer Platten-Aktuator mit AFM-Cantilever und (c) AFMCantilever mit Spitze

Resonanzen auf. Der Einsatz von kurzen piezoelektrischen PlattenAktuatoren wird jedoch durch einen kleineren vertikalen Aussteuerbereich begrenzt.

Während die mit dem AFM abzubildende Oberflächenstruktur typischerweise eine Topographie von nur wenigen Nanometern aufweist, welcher mit dem reduzierten Stellbereich problemlos gefolgt werden kann, treten durch eine meist nicht vermeidbare Verkippung der Probenoberfläche gleichzeitig große vertikale Hübe auf (einige $\mu \mathrm{m}$ ). Dieser große vertikale Aussteuerbereich muss jedoch nur für Frequenzen bis zur zehnfachen Zeilenfrequenz gewährleistet werden. Die Forderung nach einer hohen vertikalen Bandbreite kann somit auf relativ kleine (einige $100 \mathrm{~nm}$ ) vertikale Auslenkungen beschränkt werden

Das Konzept eines dual aktuierten Systems setzt an genau diesem Punk an und verwendet für die Nachführung der Probentopografie einen kurzen und schnellen piezoelektrischen Platten-Aktuator (Abb. 5b), welcher den hochfrequenten Auslenkungen folgen kann (Sulchek et al., 1999; Schitter, 2009; Kuiper, Schitter, 2010). Für die Kompensation von niederfrequenten Auslenkungen mit großen Amplituden kommt ein herkömmlicher piezoelektrischer Röhrchenaktuator (Abb. 5a) zum Einsatz.
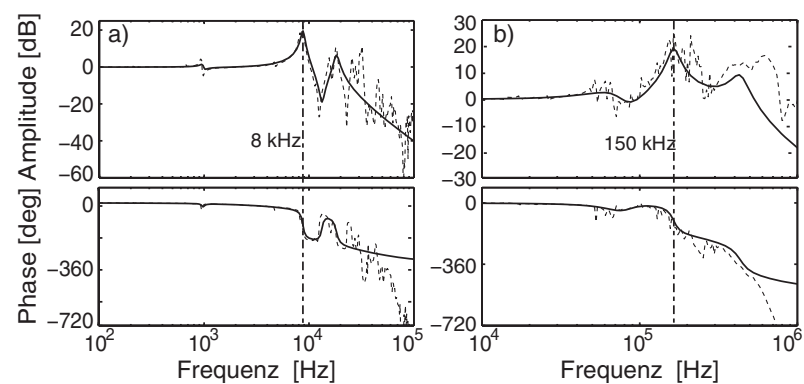

Abb. 6. Gemessene (strichliert) und modellierte Frequenzgänge (durchgezogen) des piezoelektrischen Röhrchenscanners (a) und piezoelektrischen Platten-Aktuators (b)

Abbildung 6 zeigt den gemessenen (strichliert) und modellierten Frequenzgang $G_{1}$ des piezoelektrischen Röhrchenscanners mit einer maximalen Längenänderung von $0,5 \mu \mathrm{m}$ (Abb. 6a) sowie den Frequenzgang $G_{2}$ des piezoelektrischen Stack-Aktuators mit einer maximalen Längenänderung von $0,5 \mu \mathrm{m}$ (Abb. 6b). Die erste mechanische Resonanzfrequenz des Röhrchenscanners in vertikaler Richtung liegt bei $8 \mathrm{kHz}$, die des Platten-Aktuators bei $150 \mathrm{kHz}$. Die Grenzfrequenzen für die Positionsregelung wurden mit $6 \mathrm{kHz}$ und $40 \mathrm{kHz}$ unter den entsprechenden Resonanzen festgelegt. Weiters sind in Abb. 6 die Frequenzgänge der Modelle 7ter Ordnung gezeigt, welche auf Basis mehrerer Messungen mit unterschiedlichen Cantilevern erstellt wurden. Diese Modelle wurden in Verbindung mit den charakterisierten Messunsicherheiten zur Implementierung einer modellbasierten Regelung verwendet (Kuiper, Schitter, 2010).

Bei der Realisierung eines dual aktuierten Systems ist vor allem die Wahl einer geeigneten Übergabefrequenz zwischen den beiden Aktuatoren von entscheidender Bedeutung (Schroeck, Messner, 1999), da eine ungünstige Wahl zu einem konkurrierenden Betrieb beider Aktuatoren führen kann (Kuiper, Schitter, 2010). Die Optimierung der Regler kann nicht unabhängig erfolgen, da jede Transferfunktion (Gleichungen 5 und 6) durch die Rückkopplung eine Abhängigkeit von beiden Reglern $K_{1}$ und $K_{2}$ sowie den Strecken $G_{1}$ und $G_{2}$ aufweist.
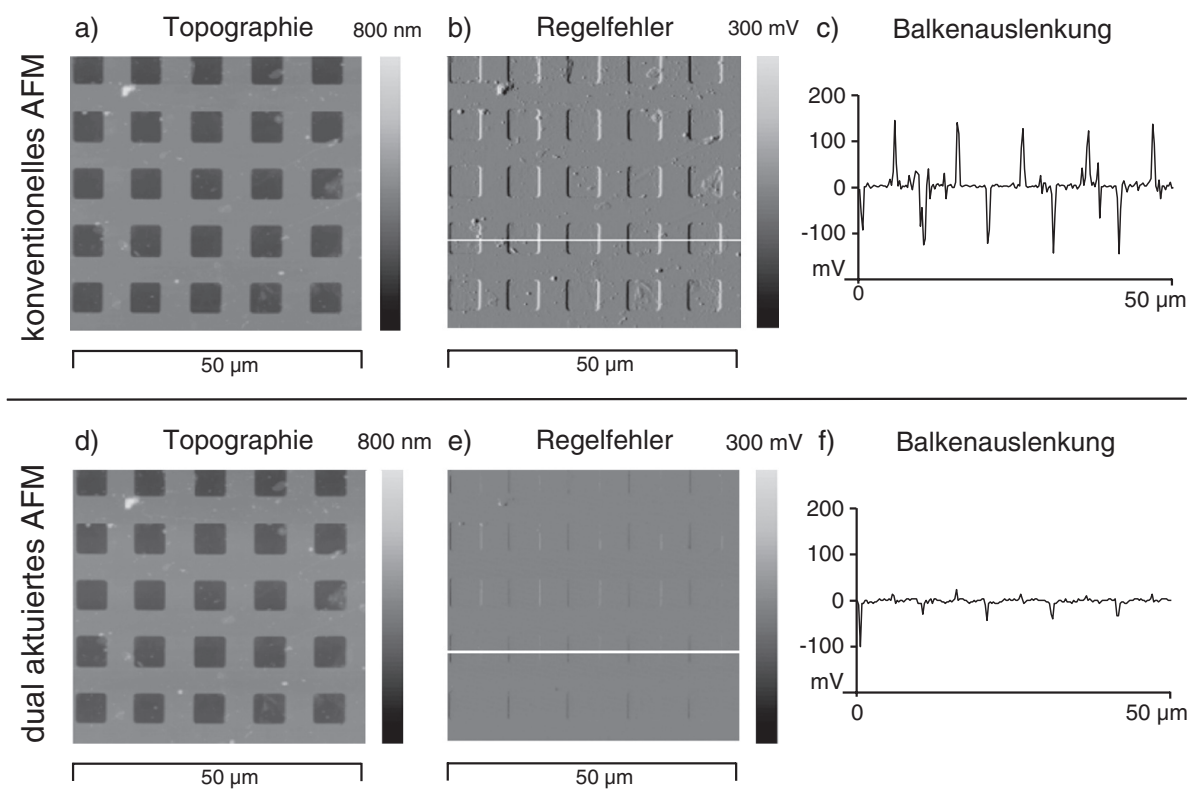

Abb. 7. Vergleich der Messung einer Kalibrationsprobe mit einem konventionellem AFM (a-c) und einem dual aktuierten AFM (d-f). Der Regelfehler, gegeben durch die Auslenkung des Cantilevers, wird im Falle des dual aktuierten AFMs (e und f) signifikant reduziert 


$$
\begin{aligned}
& T_{1}(s)=\frac{G_{1} K_{1}}{1+G_{1} K_{1}+G_{2} K_{2}} \\
& T_{2}(s)=\frac{G_{2} K_{2}}{1+G_{1} K_{1}+G_{2} K_{2}}
\end{aligned}
$$

Die gesamte Transferfunktion ergibt sich zu $T_{\text {ges }}(s)=T_{1}(s)+T_{2}(s)$. Stabilitätsprobleme durch eine allfällige Sättigung des kurzen Aktuators an hohen Topographiekanten oder bei der Näherung der Spitze an die Probe müssen durch ein geeignetes Anti-wind-up-Konzept vermieden werden (Kuiper, Schitter, 2011).

Abbildung 7 zeigt den Vergleich des konventionellen AFMs $(a-c)$ mit dem dual aktuierten AFM (d-f) bei Aufnahme einer Kalibrationsprobe, die quadratische Löcher mit einer Tiefe von $200 \mathrm{~nm}$ bei einer lateralen Periode von $10 \mu \mathrm{m}$ aufweist. In 7a) und d) ist die Topographie, welche der vertikalen Auslenkung der Aktuatoren entspricht, dargestellt, in 7b) und e) der verbleibende Regelfehler, welcher der vertikalen Auslenkung des Cantilevers entspricht. Das Profil der Auslenkung des Cantilevers über den in 7b) und e) gekennzeichneten Linien ist in 7c) und f) dargestellt. Gegenüber dem konventionellen AFM, bei dem die maximale Auslenkung des Cantilevers ein Sensorsignal von ca. $150 \mathrm{mV}$ zeigt, weist das dual aktuierte System an den Kanten des Kalibrationsgitters eine wesentlich geringere Auslenkung des Cantilevers von lediglich $40 \mathrm{mV}$ auf.

Die signifikante Reduktion des verbleibenden Regelfehlers bedeutet gleichzeitig eine Reduktion der maximal auftretenden Abbildungskräfte und erlaubt somit deutlich schnellere Messungen, ohne die Spitze oder Probe zu beschädigen.

\section{Zusammenfassung}

Dieser Beitrag zeigt, wie die Abbildungsqualität und Messgeschwindigkeit in der Rasterkraftmikroskopie mittels moderner Regelungstechnik und mechatronischer Systemintegration signifikant gesteigert werden kann.

Durch aktive Dämpfung des Aktuators in X- und Y-Richtung, basierend auf dem Self-sensing-Effekt, wurde die erste Resonanzüberhöhung des Röhrchenaktuators in der jeweiligen Scan-Richtung signifikant reduziert. Rein durch Anpassung des elektronischen Designs und Einbettung des Aktuators in eine kapazitive Brückenschaltung, ohne den Einsatz von expliziten Positionssensoren, konnten eine Dämpfung der Resonanz in der jeweiligen Scan-Richtung um $18 \mathrm{~dB}$ sowie eine Reduktion des Übersprechens zwischen den beiden Scan-Richtungen von mehr als $30 \mathrm{~dB}$ erreicht und somit oszillationsbedingte Abbildungsfehler minimiert werden.

Um die Bandbreite des Regelkreises zur Messung der Probentopographie zu erhöhen, ohne dabei den Stellbereich des Aktuators zu beeinträchtigen, wurde ein dual aktuiertes AFM-System, bestehend aus einem schnellen Platten-Aktuator in Kombination mit einem Röhrchenscanner, implementiert. Die höhere Bandbreite des dual aktuierten Systems führt zu einer signifikanten Reduktion des verbleibenden Regelfehlers und erlaubt somit, mit geringeren Interak- tionskräften zwischen der Messspitze und Probenoberfläche abzubilden und die Messgeschwindigkeit entsprechend zu erhöhen, ohne dabei die Spitze oder Probe durch hohe Abbildungskräfte zu beschädigen.

Literatur

Alexander, S., Hellemans, L., Marti, O., Schneir, J., Elings, V., Hansma, P., Longmire, M., Gurley, J. (1989): An atomic-resolution atomic-force microscope implemented using an optical lever. J. Appl. Phys., 65: 164.

Bhikkaji, B., Ratnam, M., Fleming, A. J., Moheimani, S. O. R. (2007): High performance control of piezoelectric tube scanners. IEEE Trans. Control Syst. Technol. 15: 853-866.

Binnig, G., Quate, C., Gerber, C. (1986): Atomic force microscope. Phys. Rev. Lett., 56 (9): 930-933.

Binnig, G., Smith, D. (1986): Single-tube three-dimensional scanner for scanning tunneling microscopy. Rev. Sci. Instrum., 57: 1688-1698.

Croft, D., Shed, G., Devasia, S. (2001): Creep, hysteresis, and vibration compensation for piezoactuators: Atomic force microscopy applications. AMSE J. Dyn. Syst. Meas. Control, 123: 35-43.

Dosch, J., Inman, D., Garcia, E. (1992): A self-sensing piezoelectric actuator for collocated control. J. Intell. Mater. Syst. Struc., 3: 166-185.

Fleming, A., Moheimani, S. (2006): Sensorless vibration suppression and scan compensation for piezoelectric tube nanopositioners. IEEE Trans. Control Syst. Technol., 14: 33-44.

Hansma, P., Schitter, G., Fantner, G., Prater, C. (2006): High speed atomic force microscopy. Science, 314: 601-602.

Kuiper, S., Fleming, A., Schitter, G. (2010): Dual actuation for high speed atomic force microscopy. In: Proc. IFAC Mechatronics Conf.

Kuiper, S., Schitter, G. (2009): Self-Sensing Actuation and Damping of a Piezoelectric Tube Scanner for Atomic Force Microscopy. European Control Conf., 2009.

Kuiper, S., Schitter, G. (2010): Active damping of a piezoelectric tube scanner using self-sensing piezo actuation. Mechatronics, 20: 656-665.

Kuiper, S., Schitter, G. (2011): Model-based feedback controller design for dual actuated atomic force microscopy. Mechatronics (in press).

Picco, L., Bozec, L., Ulcinas, A., Engledew, D., Antognozzi, M., Horton, M., Miles, M. (2007): Breaking the speed limit with atomic force microscopy. Nanotechnology, 18 (044030): 4

Rifai, O., Youcef-Toumi, K. (2001): Coupling in piezoelectric tube scanners used in scanning probe microscope. Proc. Amer. Control. Conf., 4: 3251-3255.

Salapaka, S., Sebastian, A., Cleveland, J., Salapaka, M. (2002): High bandwidth nano-positioner: a robust control approach. Rev. Sci. Instrum., 73: 3232.

Sarid, D. (1994): Scanning force microscopy: with applications to electric, magnetic and atomic forces. Oxford University Press, USA.

Schitter, G. (2009): Improving the Speed of AFM by Mechatronic Design and Modern Control Methods. Tech. Mess., 76 (5): 266-273.

Schitter, G. Stemmer, A. (2004): Identification and open-loop tracking control of a piezoelectric tube scanner for high-speed scanning-probe microscopy. IEEE Trans. Control Syst. Technol., 12: 449-454.

Schroeck, S., Messner, W. (1999): On controller design for linear time-invariant dual-input singleoutput systems. Proc. Amer. Control Conf., vol. 6.

Sebastian, A., Salapaka, S. (2005): Design methodologies for robust nano-positioning. IEEE Trans. Control Syst. Technol., 13 (6): 868-876.

Sulchek, T., Minne, S., Adams, J., Fletcher, D., Atalar, A., Quate, C., Adderton, D. (1999): Dual integrated actuators for extended range high speed atomic force microscopy. Appl. Phys. Lett., 75: 1637-1639.

Tamer, N., Dahleh, M. (1994): Feedback control of piezoelectric tube scanners. In: Proc. 33rd IEEE Conf. Decis. Control, 2: 1826-1831. 


\section{Autoren}

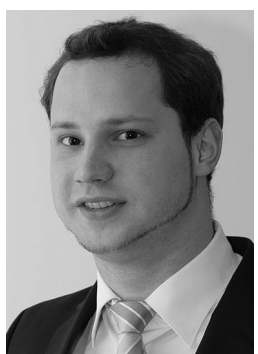

\section{Jürgen Steininger}

ist als Assistent am Institut für Automatisierungs- und Regelungstechnik (ACIN) der Technischen Universität Wien tätig. Nach dem Bakkalaureatsstudium der Elektrotechnik schloss er 2010 das Masterstudium der Mikroelektronik an der TU Wien mit Auszeichnung ab. Nach einem erfolgreichen FFG-Projekt zur kapazitiven Nahfeldkommunikation beschäftigt er sich momentan im Zuge seiner Dissertation mit der Sensorik in der Mikro- und Nanotechnologie.

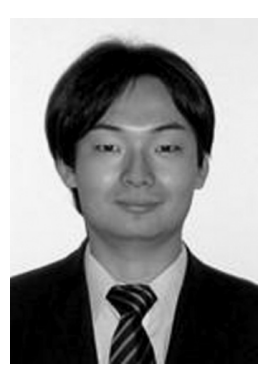

\section{Shingo Ito}

erhielt seinen Bachelorabschluss von der Chiba University in Japan im Jahr 2004. Seinen M.Sc. erhielt er von der Universität Toronto in Kanada im Jahre 2007. Seit 2011 forscht er als Assistent im Zuge seiner Dissertation an der Technischen Universität Wien an HochpräzisionsPositioniersystemen.

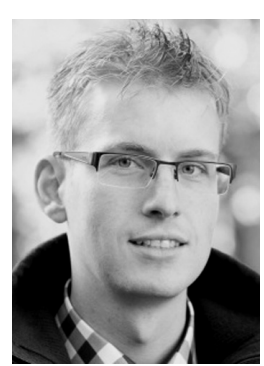

\section{Stefan Kuiper}

erhielt seinen Masterabschluss in Mechanical Engineering im Jahre 2007 von der University of Twente in den Niederlanden. Momentan arbeitet er an seiner Dissertation an der Delft University of Technology in den Niederlanden im Bereich Mechatronik und Regelungstechnik für hochpräzise Positioniersysteme.

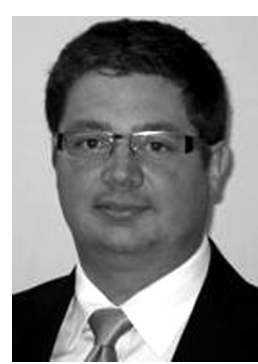

\section{Georg Schitter}

absolvierte das Studium Elektrotechnik, Studienzweig Prozesstechnik, an der Technischen Universtität Graz (2000) sowie ein Nachdiplomstudium in Informationstechnik (2004) und ein Doktoratsstudium in Nanotechnologie (2004) an der ETH Zürich. Von 2004 bis 2006 forschte er an der University of California Santa Barbara (UCSB). 2006 wurde er als Assistenzprofessor an die TU Delft, Niederlande, berufen, wo er 2009 zum Associate Professor ernannt wurde. Seit Juli 2010 leitet Georg Schitter den Lehrstuhl für "Industrielle Automationstechnik" am Institut für Automatisierungsund Regelungstechnik der TU Wien.

Die Forschungsinteressen von Georg Schitter befassen sich mit der Entwicklung integrierter Systemlösungen inklusive neuer system- und regelungstechnischer Methoden für bildgebende mechatronische Systeme und für präzisionstechnologische Anwendungen bis in den Nanometerbereich, insbesondere für Fertigungssysteme und In-Prozess-Messtechnik sowie für die Materialund Biowissenschaften. 